\title{
CFD Fuel Slosh Modeling of Fluid-Structure Interaction in Spacecraft Propellant Tanks with Diaphragms
}

\author{
Dillon J. Sances ${ }^{1}$ and Sathya N. Gangadharan ${ }^{2}$ \\ Embry-Riddle Aeronautical University, Daytona Beach, FL, 32114 \\ James E. Sudermann ${ }^{3}$ and Brandon Marse $\mathbf{l}^{4}$ \\ NASA, Kennedy Space Center, FL, 32899
}

\begin{abstract}
Liquid sloshing within spacecraft propellant tanks causes rapid energy dissipation at resonant modes, which can result in attitude destabilization of the vehicle. Identifying resonant slosh modes currently requires experimental testing and mechanical pendulum analogs to characterize the slosh dynamics. Computational Fluid Dynamics (CFD) techniques have recently been validated as an effective tool for simulating fuel slosh within free-surface propellant tanks. Propellant tanks often incorporate an internal flexible diaphragm to separate ullage and propellant which increases modeling complexity. A coupled fluid-structure CFD model is required to capture the damping effects of a flexible diaphragm on the propellant. ANSYS multidisciplinary engineering software employs a coupled solver for analyzing two-way Fluid Structure Interaction (FSI) cases such as the diaphragm propellant tank system. Slosh models generated by ANSYS software are validated by experimental lateral slosh test results. Accurate data correlation would produce an innovative technique for modeling fuel slosh within diaphragm tanks and provide an accurate and efficient tool for identifying resonant modes and the slosh dynamic response.
\end{abstract}

\section{Introduction}

T IQUID propellants can induce motions within partially filled propellant tanks known as fuel slosh. This fluid Lehavior occurs primarily at the liquid surface and oscillates, exerting forces on the tank structure walls and changing inertia. External torques and resonant modes can excite the fuel and amplify slosh behavior, causing rapid energy dissipation. ${ }^{1}$ One method used to dampen fuel slosh while simultaneously ensuring no gas bubbles enter the fuel lines is to add a flexible elastomeric diaphragm between the gas and liquid inside the tank. Simple, free-surface fuel slosh can be readily modeled using Computational Fluid Dynamics(CFD) but adding a flexible diaphragm to the CFD model has been problematic. Recent breakthroughs in multidisciplinary Fluid Structure Interaction (FSI) solvers have developed tools to analyze systems in which both liquid and solid components affect the system dynamics. This capability is essential for calculating the effects of fuel slosh in tanks with non-rigid internal propellant management structures such as diaphragms. Using CFD-FSI solvers to model fuel slosh can increase initial spacecraft design efficiency by reducing experimental testing and associated costs.

Recent and historical space missions have been subjected to unexpected fuel slosh effects, some resulting in mission failure. In 2007, a Falcon-1 launch vehicle failed to reach it's targeted orbit due to fuel slosh oscillations. The nozzle of the upper stage was bumped during a separation event, which excited onboard propellant and generated slosh waves. The onboard control system interacted with these slosh waves in an unexpected fashion, amplifying their intensity and causing propellant starvation of the second stage engine as the fuel sloshed back and forth around the fuel pickup at the end of the burn.

Launch vehicles and spinning space systems are sensitive to changes in inertia and attitude control mechanisms. Energy dissipation from the slosh can cause spinning spacecraft to wobble and become unstable since the majority of inertia in many spacecraft is propellant. This wobbling/precession about the spin axis is known as nutation. Viscous effects associated with these oscillations cause the spacecraft's rotational kinetic energy to be converted

\footnotetext{
${ }^{1}$ Graduate Student, Department of Mechanical Engineering, AIAA Student Member.

${ }^{2}$ Professor, Department of Mechanical Engineering, AIAA Associate Fellow.

${ }^{3}$ Controls Analyst, NASA Launch Services Program.

${ }^{4}$ Controls Analyst, NASA ANALEX Corporation, AIAA Member.
} 
into molecular kinetic energy. ${ }^{2}$ Since angular momentum remains constant, internal torques created by the liquid oscillations cause rotational kinetic energy to change, resulting in nutation growth.

The energy dissipation rate of fuel slosh can be defined by a Nutation Time Constant (NTC). ${ }^{3}$ Free-surface fuel slosh involves only viscous damping and occurs in propellant tanks with no internal damping structures. For a freesurface system, energy dissipates linearly and the NTC remains constant. For a spinning system, the NTC defines the exponential rate of nutation growth.

The implementation of internal tank structures introduce non-linear liquid damping into the system and the NTC no longer remains constant. Propellant Management Devices (PMD's) such as diaphragms are often integrated to ensure a steady, gas bubble free flow of propellant and to dampen fluid slosh. A diaphragm is a flexible structure mounted at the center of the tank which couples the liquid surface. The device conforms to the liquid as propellant is consumed; assisted by expelling pressurant into the empty volume of the tank. Various diaphragm geometries are shown in Fig. 1.

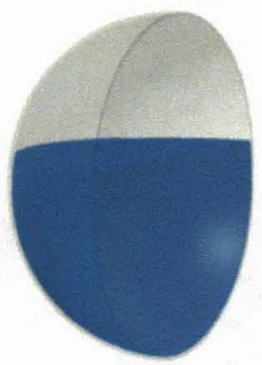

a)

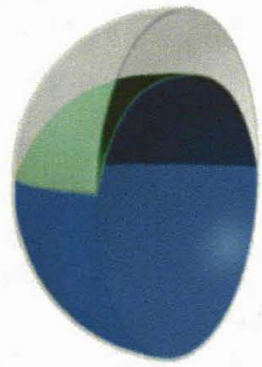

b)

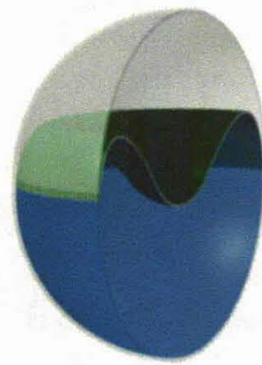

c)

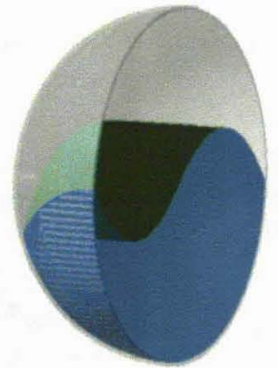

d)

Figure 1. Various diaphragm shapes conforming to the liquid surface.
a) Free-Surface Tank
b) "Mountain" shaped diaphragm

c) "Crater" shaped diaphragm

d) "Yin-yang" shaped diaphragm

Diaphragm devices consist of a thin elastomeric material, which is flexible enough to deform to the liquid surface, but can be stiff enough to hold a specific shape under certain excitations. A photograph of a spacecraft ground test propellant tank with a diaphragm in the crater shape is shown in Fig. 2.

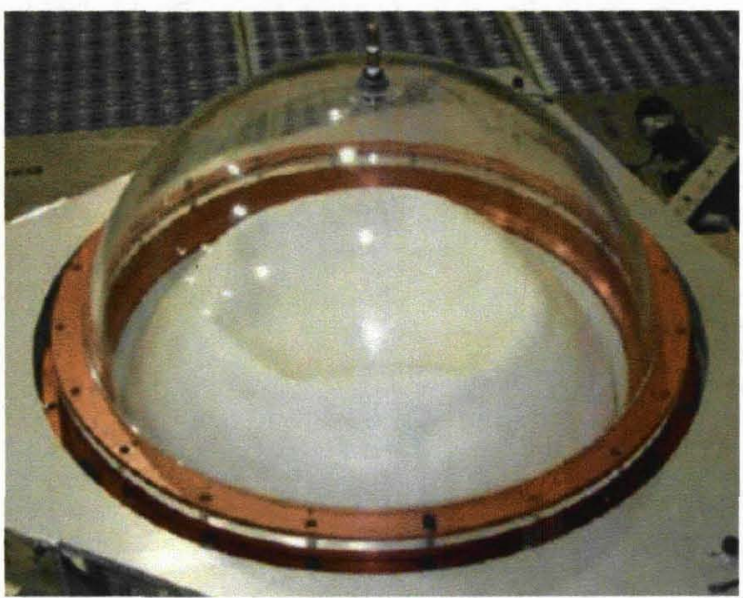

Figure 2. Example of a crater shaped diaphragm at $60 \%$ fill level.

Free-surface fuel slosh CFD models have been validated by prior research and have provided a new approach to analyzing slosh dynamics. Figure 3 illustrates free-surface slosh using CFD. The model incorporates a Volume of Fluids (VOF) technique, which captures the interface between two fluids; the liquid propellant and the gas pressurant. CFD models can simulate the periodic motion of fuel slosh within spacecraft propellant tanks. Another great advantage of using CFD is the ability to vary parameters such as gravity, tank geometry, PMD type, and viscosity, which would be difficult, and in some cases, impossible to imitate in a laboratory. 


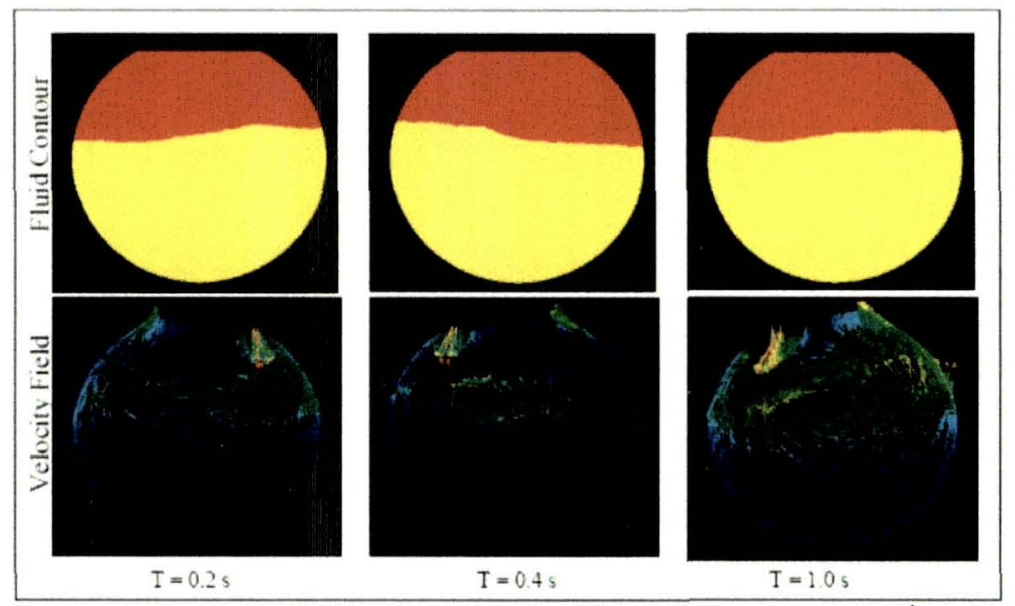

Figure 3. CFD simulation of free-surface fuel slosh. ${ }^{4}$

Multi-disciplinary CFD software is an innovative tool for simulating slosh dynamics in propellant tanks with a diaphragm. ANSYS' MultiField Solver (MFX) couples a fluid-physics solver and a structures solver, allowing full two-way Fluid Structure Interaction (FSI) representation of the diaphragm tank system. Two-way FSI is required for modeling nonlinear slosh. The fluid conforms to the diaphragm while the diaphragm simultaneously dampens the liquid. The MFX solver switches between a structures solver, ANSYS Mechanical, and a fluids solver, ANSYS CFX, at each time step by providing feedback of forces and displacements between the two modules. In our research, CFD models are validated by comparing results to experimental test data and to models developed using MATLAB SimMechanics software. SimMechanics mechanical models use a simple pendulum analog to characterize fuel slosh.

\section{Experimental Data Collection}

Embry-Riddle Aeronautical University (ERAU) operates a propellant slosh testing laboratory and lateral slosh experimental setup as shown in Fig. 4. Experimental slosh data is acquired to validate CFD generated models. An 8" spherical propellant tank is suspended by wires allowing it move back and forth, similar to a pendulum. Data acquisition consists of a force transducer fixed to the side of the tank which measures the reaction force from propellant sloshing against the tank wall in the lateral axis. A linear actuator is used to excite the fluid at resonant frequencies and oscillate the liquid to measure the force response at the tank wall. Typical spacecraft liquid propellants such as hydrazine are highly toxic and difficult to handle in a laboratory. Water is substituted for hydrazine propellant due to similar fluid properties.

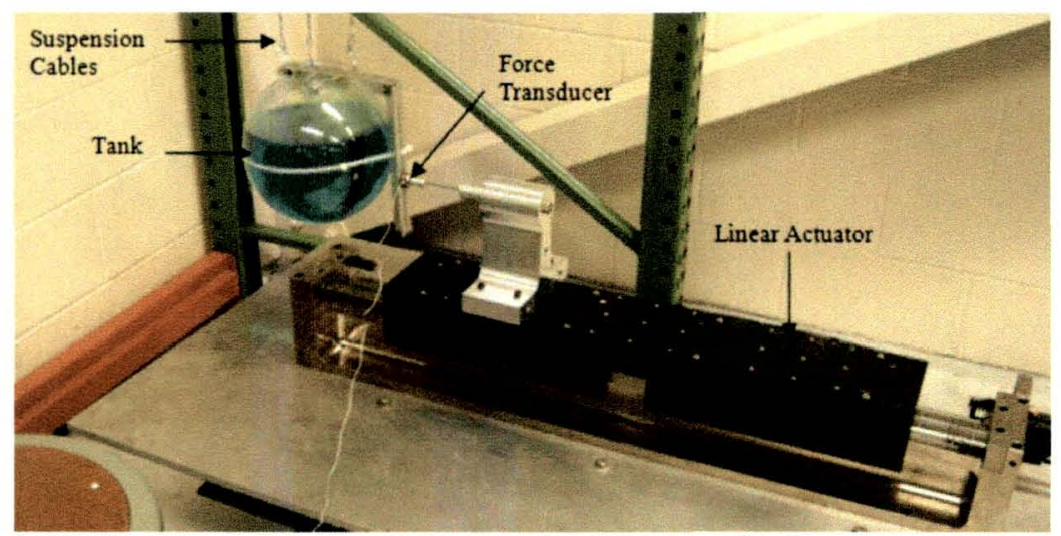

Figure 4. Fuel slosh experimental setup at ERAU (Free-Surface test scenario shown).

An 8" diaphragm propellant tank was excited at a resonant frequency to measure the tank wall reaction force. This test scenario incorporated a flexible diaphragm at the tank centerline, which provided the structural interface between water and air. The water in the tank is fixed at a $60 \%$ volume fill level - which has been identified through experimental testing to induce maximum slosh amplitude. ${ }^{5}$ Various test data was collected for different diaphragm 
shapes, fluid materials, and fill fractions, however only the $60 \%$ case of water with a crater diaphragm was investigated for CFD-FSI model validation. The damping results are shown in Fig.5.

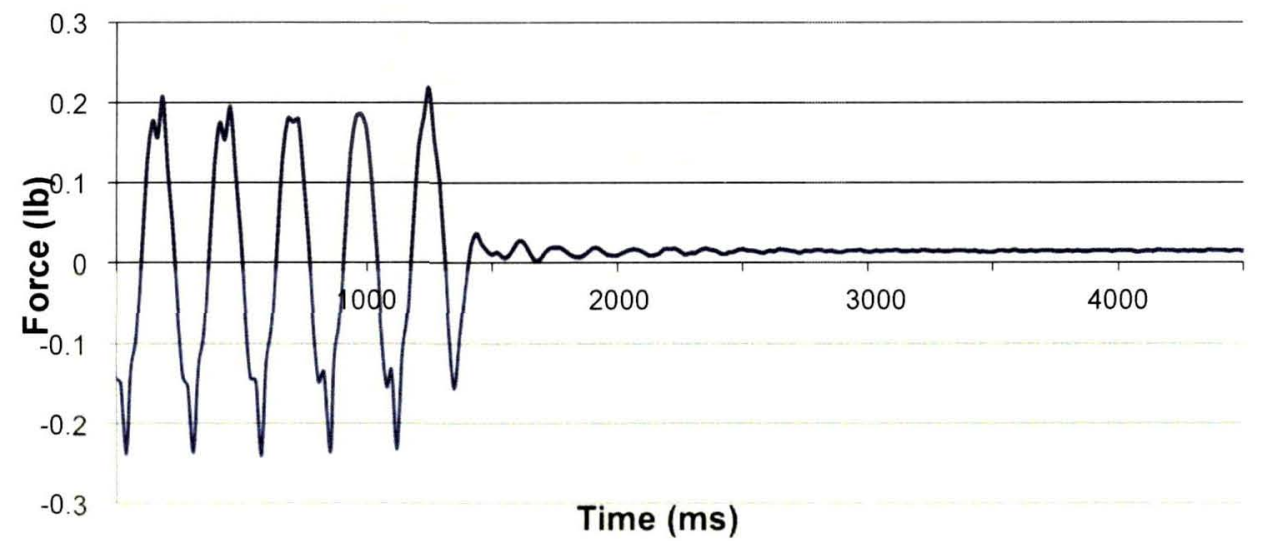

Figure 5. Damping of water $60 \%$ with diaphragm.

III. CFD Modeling of Lateral Fuel slosh with FSI

The diaphragm propellant tank system is modeled using two-way CFD-FSI software ${ }^{7}$. The ANSYS MFX solver is utilized to produce an FSI fuel slosh solution by coupling ANSYS CFX and ANSYS Mechanical solvers. Forces exerted on the diaphragm from the sloshing liquid surface interact with the diaphragm nonlinearly.

To demonstrate a CFD-FSI system, a simplified slosh scenario is initially considered. A 12" spherical tank with $50 \%$ water and $50 \%$ air is considered with a flat diaphragm mounted at the tank centerline. The fluid domain is locally initiated with a $1 \mathrm{~m} / \mathrm{s}$ lateral velocity for excitation and a transient FSI analysis is solved. The illustration in Fig. 6 shows post-processing fluid pressure results of this simplified FSI model with amplified deformation. The diaphragm displacement is shown in Fig. 7 with amplified deformation as well. The liquid oscillates along a lateral axis and dampens rapidly from the diaphragm boundary. The fluid (water) is incompressible, causing the fluid to build up at the tank wall and air replaces the volume opposite to the bulk slosh wave, which produces a yin-yang shape. Figures 6 and 7 are not very realistic geometries for a diaphragm PMD but it shows the interaction between the water, diaphragm, and air.

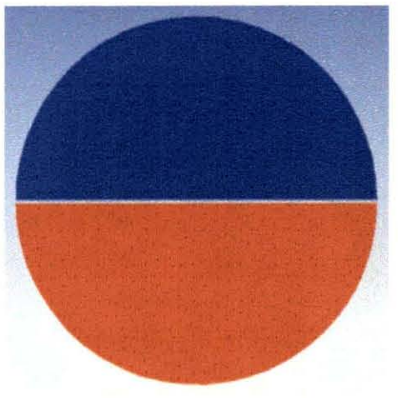

$\mathrm{t}=0 \mathrm{~s}$

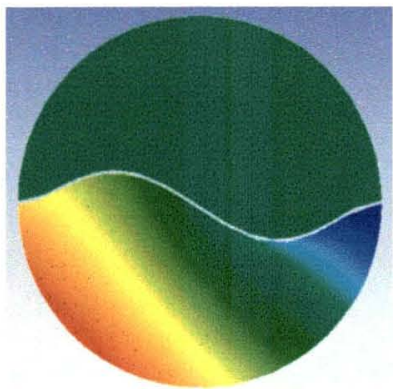

$\mathrm{t}=0.1 \mathrm{~s}$

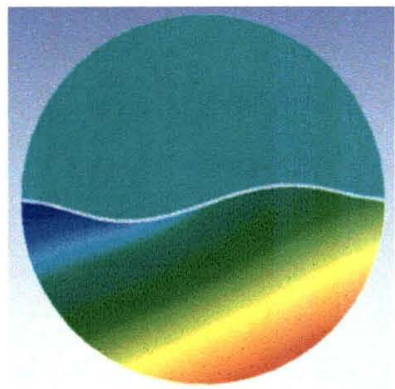

$\mathrm{t}=0.2 \mathrm{~s}$

Figure 6. Transient water pressure results using a CFD-FSI solver for diaphragm fuel slosh system.

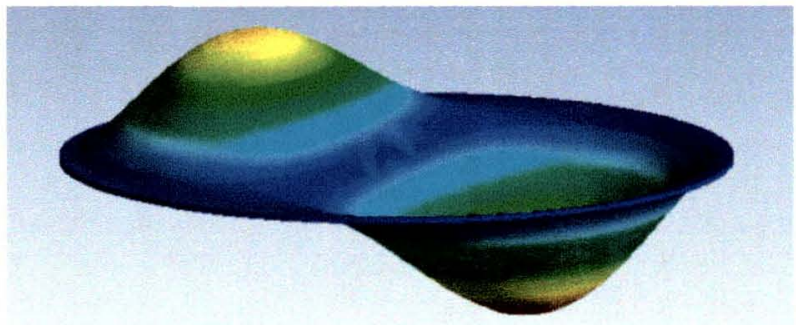

Figure 7. Amplified diaphragm displacement at $t=0.1 \mathrm{~s}$.

4

American Institute of Aeronautics and Astronautics 


\section{CFD-FSI Fuel Slosh Model with Crater Diaphragm}

A CFD fuel slosh model representing the experimental diaphragm propellant tank is now generated for comparison and validation. The model consists of an $8 "$ spherical diaphragm tank filled to $60 \%$ with water. The diaphragm geometry resembles a "crater" shape and is flexible, yet stiff enough to hold its shape under testing conditions and the CFD model geometry must be consistent with that of the experimental tank geometry. Water is substituted for hydrazine liquid rocket fuel, and air is utilized as the pressurant. As fuel depletes from the propellant tank, a pressure regulator expels a gas into the tank to equalize the tank pressure and assist conforming the diaphragm to the liquid surface. This model incorporates exciting a constant fuel/water volume at a resonant frequency to determine the non-linear force response at the tank walls.

The ANSYS software allows users to model the system globally using the ANSYS Workbench module. This creates a sequential procedure to solving the two-way FSI model as shown in Fig. 8. The Workbench allows for sharing of similar geometry and solver criteria between CFX and ANSYS Mechanical. The FSI architecture requires the structural solver to be setup prior to the fluid solver. The first step embedded in the Workbench objects is to identify geometry domains and appropriately mesh them based on expected boundary conditions and physics classification.

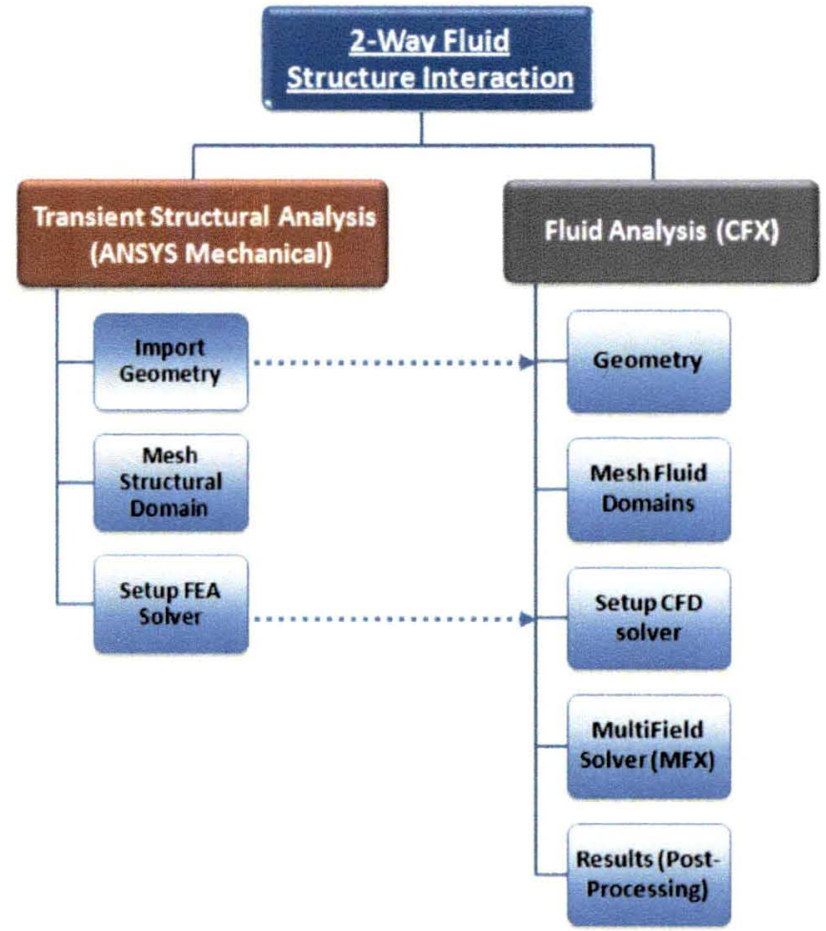

Figure 8. Two-way FSI functional diagram architecture for ANSYS Workbench module.

The diaphragm structural component is setup using ANSYS Mechanical by isolating the structural domain from fluids in the system geometry and identifying boundary conditions. ANSYS uses an automated tetrahedral mechanical meshing method to produce an optimal mesh for the structural domain as shown in Fig. 9.

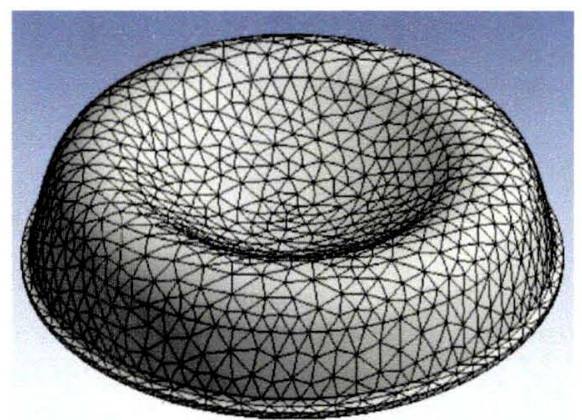

Figure 9. Mesh geometry of diaphragm structure. 
The diaphragm material is assigned to the structure domain in ANSYS Mechanical but some assumptions must also be considered to reduce solver processing time. It is important to identify material properties of the diaphragm such as density, strength, and elasticity. A crater diaphragm shape is used to form the diaphragm geometry. Diaphragms can conform to the liquid in different shapes as stated previously but different geometries can be validated in subsequent research. The diaphragm is flexible and conforms to the liquid, however it does not stretch or shrink. It merely changes shape as propellant is exerted from the tank. Therefore, the diaphragm's surface area remains constant. This simplifies structural analysis of the diaphragm because nonlinearities of the diaphragm material itself are considered negligible. The diaphragm can then be considered iso-elastic, which reduces the required material property inputs to just density, modulus of elasticity, and poisons ratio. The damping of the liquid from the coupled diaphragm is however non-linear, again confirming the need for CFD-FSI solutions.

The boundary conditions must be identified on each domain to characterize the geometry. The structural domain includes just the diaphragm crater geometry. This component consists of two boundary conditions, one constraint, and gravity, which are defined using the ANSYS Mechanical module. The outer circumferential surface of where the diaphragm mounts to the centerline of the propellant tank is constrained as a fixed support. For FSI models, fluid-structure interface boundaries must be identified to allow for coupled mesh deformation resulting from fluid motion. The top and bottom surfaces of the diaphragm are identified as the two fluid-structure interfaces; one for water and another for air. These are labeled appropriately as shown in Fig. 10 and will be referenced later when identifying fluid-interface boundaries on the fluid domains. Gravity is the only constant external acceleration on the structural domain.

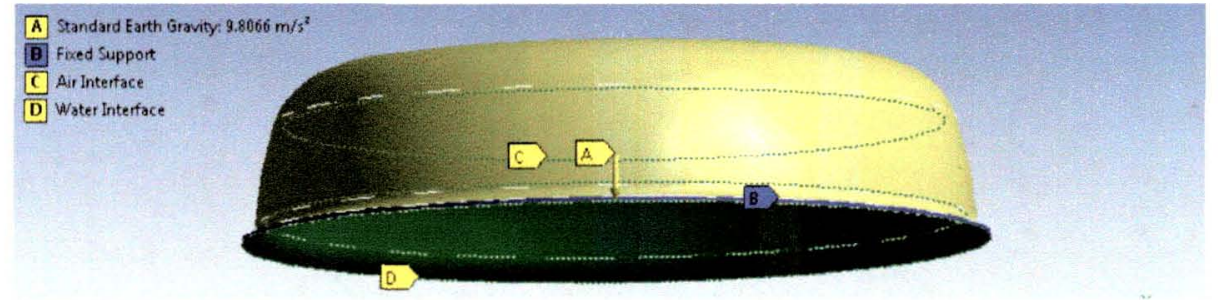

Figure 10. Fluid structure interface surface boundaries and constraints for structural domain.

The fluid domains are meshed similarly to that of the diaphragm. An automated tetrahedral mesh is generated for the fluid domains however; CFD physics are employed to comply with CFX meshing requirements. Cells close to the geometric boundaries decrease in size using fine local meshing schemes as shown in Fig. 11. Pre-processing of the fluid domains is accomplished using ANSYS CFX. This CFD module requires definition of fluid domains, boundary conditions, and initial conditions. The fluids are separated into two separate domains; air and water. For both fluids, fluid-structure boundaries must be identified for mesh displacement using MFX. The tank walls are considered a stationary boundary implying no mesh deformation. Both the water and air domains have similar boundaries and can be setup similarly.

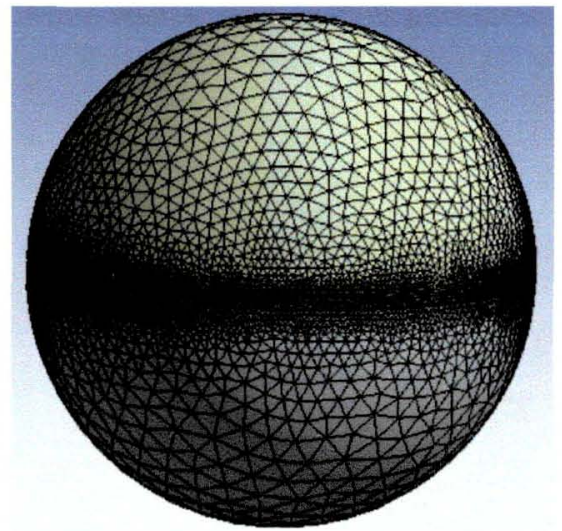

Figure 11. Mesh geometry of fluid domains.

The fluids are both easily referenced to appropriate material properties for air and water using the software material library and classified as continuous fluids. The mesh volume of each domain must first be characterized to

6

American Institute of Aeronautics and Astronautics 
account for realistic liquid motion. Since two fluid materials have added to the system, CFX requires a Volume of Fluids (VOF) method to be employed for each domain. This will be referenced when identifying initial conditions for volume fill levels of each domain. Gravity is important to generate oscillating slosh behavior, therefore the fluid volumes incorporate a buoyancy scheme. The fluid domains outer hemispherical surface is set as a wall boundary with a no-slip condition. The fluid-structure interface surfaces shown in Fig. 12 are the critical boundaries for the FSI model. The liquid surface coupling the diaphragm of each fluid domain is considered a wall boundary, but it also references the diaphragm fluid-structure interface boundaries from the ANSYS Mechanical utility. These FSI boundaries are adapted for coupled mesh displacement in CFX using the MFX solver.
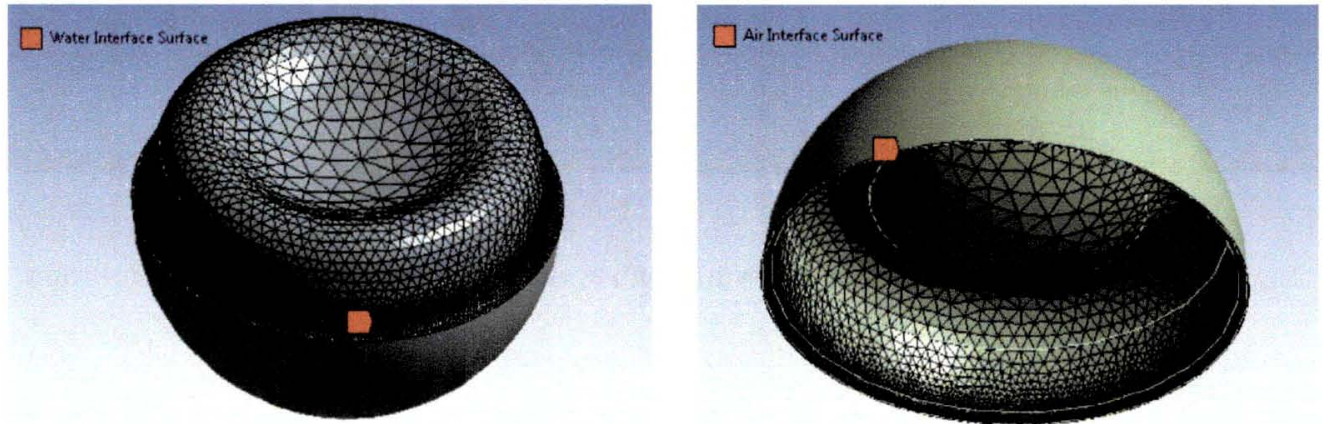

Figure 12. Fluid-structure interface surface mesh and boundaries for fluid domains.

Initial conditions for the system are acknowledged for the system on the fluid domains using ANSYS CFX. The VOF scheme implemented on the fluid domains must be initialized to specify the fluid types and volume fractions. The lower fluid domain is initialized with $100 \%$ water and the upper fluid domain on the opposite side of the diaphragm is initialized as $100 \%$ air. Fluid excitation is incorporated on the water domain to oscillate the liquid motion at a specified frequency. This is simply done by initializing the water velocity along the lateral axis, consistent with experimental lateral slosh tests. No initial conditions are required for the diaphragm structural domain.

After identifying boundaries and initial conditions, the transient solver criteria and time marching must be appropriately employed to allow for MFX solver convergence. This criterion is optimized to meet solver convergence requirements. Typical CFD solvers use time steps of 0.0001 seconds, however, to reduce computational processing demands, a larger time step of 0.01 seconds is required. The simulation is then run for only a few seconds. Heat is ignored on the system and the liquid motion is also characterized as laminar. The ANSYS MFX solver calculates the coupled CFD-FSI solution and results are analyzed using ANSYS' embedded Post-Processing utility.

\section{Results}

Transient CFD-FSI post-processing results are shown in Figs. 13 and 14. Figure 13 shows water pressure relative to the pressure domains. Using amplified deformation, the fluid's effect on the diaphragm is clearly shown as the slosh oscillates laterally across the tank. The fluid motion is dampened due to the existence of the diaphragm boundary coupling the liquid surface.

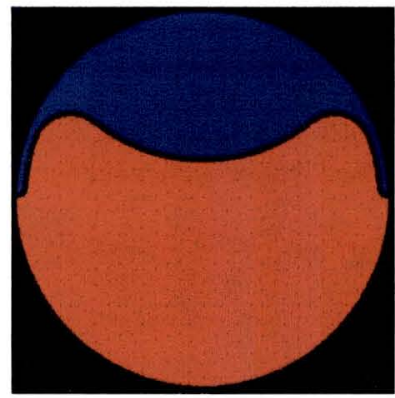

$t=0$ s

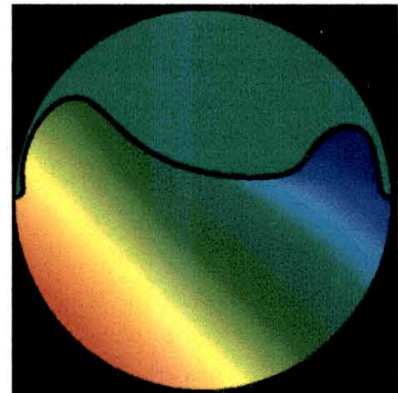

$\mathrm{t}=0.1 \mathrm{~s}$

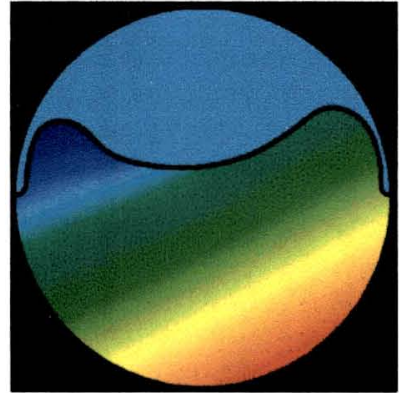

$\mathrm{t}=0.2 \mathrm{~s}$

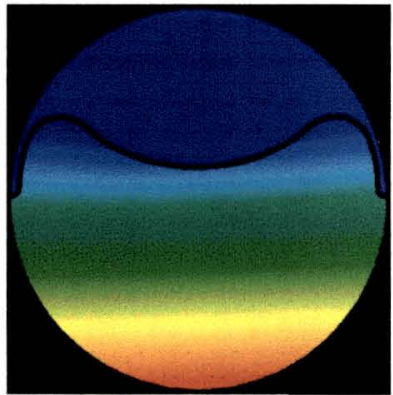

$\mathrm{t}=0.3 \mathrm{~s}$

Figure 13. Transient water pressure results from CFD-FSI fuel slosh solution. 
Figure 14 is a 3D representation of the diaphragm mesh displacement. Again, it is clearly shown with amplified deformation, that the fluid-structure interaction exists on the diaphragm and the deformation is consistent with the liquid motion beneath it.

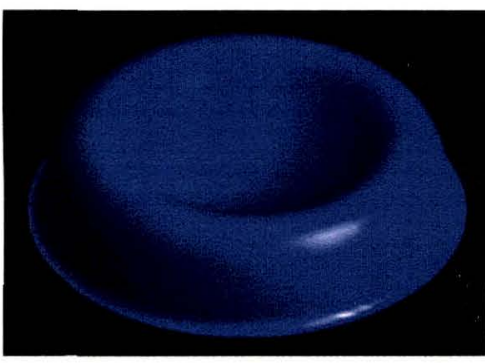

$\mathrm{t}=0 \mathrm{~s}$

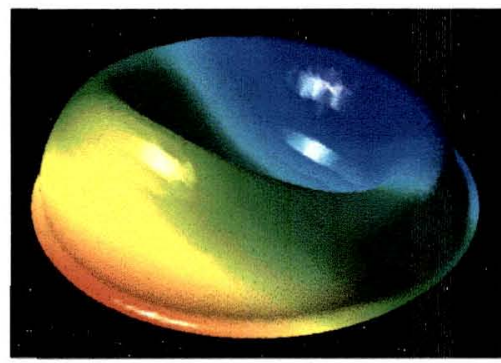

$\mathrm{t}=0.1 \mathrm{~s}$

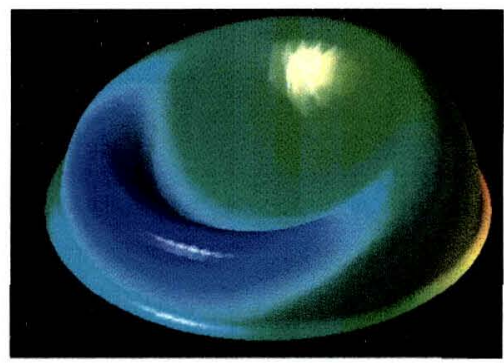

$\mathrm{t}=0.2 \mathrm{~s}$

Figure 14. Amplified diaphragm deformation results from CFD-FSI fuel slosh solution.

The tank wall pressure along the lateral excitation axis produces a sinusoidal response which characterizes the slosh damping. Figure 15 plots the wall pressure over time. The following figure clearly shows that the fluid behavior dampens out very rapidly due to the coupled diaphragm PMD

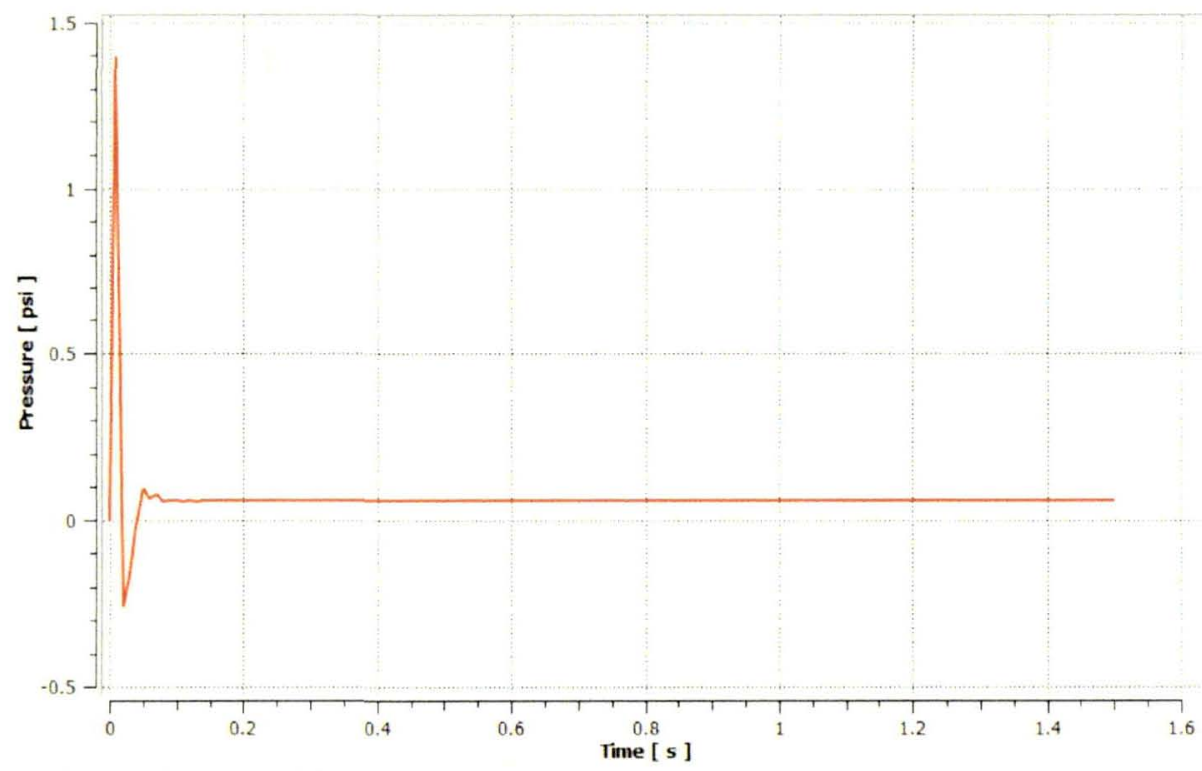

Figure 15. CFD-FSI transient pressure results at the tank wall boundary.

\section{Conclusions}

The CFD-FSI modeling approach demonstrates an innovative method for modeling fuel slosh within propellant tanks outfitted with a diaphragm. The ANSYS engineering tools provide a graphical interface to construct, mesh, and solve a diaphragm tank system using two-way FSI. Comparisons of experimental and computational results indicate that non-linear slosh dynamics can be captured using CFD methods. The CFD-FSI model produced expected fluid slosh behavior, physically consistent with oscillating slosh. Further optimization of this model and initial conditions would decrease error; however the given results were sufficient to determine if the CFD-FSI approach is an effective fuel slosh modeling technique.

This investigation confirms the capability of utilizing CFD-FSI models to parameterize fuel slosh dynamics, however, additional testing and validation is required utilizing alternate slosh scenarios to fully verify the solver capability. These scenarios include varying the liquid fill level, spherical vs. cylindrical tank geometries, and different fluid materials. The advantage of CFD is the ability to virtually change system conditions and simulate scenarios that would be difficult to reproduce experimentally. 


\section{Acknowledgments}

The authors would like to thank the Launch Services Program at NASA Kennedy Space Center for their continuous help and support for this project. Thanks to Embry-Riddle Aeronautical University for technical resources, facilitation, and experimental testing. Special thanks to the NASA Graduate Student Researchers Program (GSRP) which supports this research and development.

\section{References}

${ }^{1}$ Dodge, F.T., Unruh, J.F., Green, S.T., Cruse, M.W., "A Mechanical Model of Liquid Inertial Waves for Use with Spinning Craft," Fluid Transients, FED-Vol. 198/PVP-Vol. 291, ASME 1994.

${ }^{2}$ Hubert, Carl, "Behavior of Spinning Space Vehicles with Onboard Liquids," Technical Report B3007, Hubert Astronautics, Purcellville, Virginia, August 2003.

${ }^{3}$ Schlee, K., Gangadharan, S.N., Ristow, J., Sudermann, J., Walker, C., and Hubert, C., "Modeling and Parameter Estimation of Spacecraft Fuel Slosh," 29th Annual AAS Guidance and Control Conference, Paper\# AAS-06-027, American Astronautical Society, Rocky Mountain Section, Breckenridge, Colorado, 2006.

${ }^{4}$ Marsell, B., A Computational Fluid Dynamics Model for Spacecraft Liquid Propellant Slosh, MS Thesis, Embry-Riddle Aeronautical University, Daytona Beach, Florida 2009.

${ }^{5}$ Chatman, Y., "Mechanical Analog Approach to Parameter Estimation of Lateral Spacecraft Fuel Slosh," $49^{t h}$ AIAA/ASME/ASCE/AHS/ASC Structures, Structural Dynamics, and Materials Conference, 2008.

${ }^{6}$ Chatman, Y., Modeling and Parameters Estimation of Spacecraft Lateral Fuel Slosh, MS Thesis, Embry-Riddle Aeronautical University, Daytona Beach, Florida, 2006.

${ }^{7}$ ANSYS 12.0, 2009, ANSYS Inc, http://www.ANSYS.com 